\title{
Study and Application of Stability of Natural Organic Polymer Coagulants
}

\author{
Xiangjun Meng ${ }^{1}$, and Ying $\mathrm{Fu}^{1, *}$ \\ ${ }^{1}$ School of Civil Engineering and Architecture, University of Jinan, Jinan 250022, China
}

\begin{abstract}
The natural organic polymer coagulants have attracted much attention due to their safety, nontoxicity, good biodegradability and abundant sources of raw materials. However, the instability of coagulants has restricted their further development and application. In this paper, the main types of coagulants, the research of unstable performances and improvement measures are reviewed. Meanwhile, the applications in water treatment are introduced. Finally, the development prospects of coagulants are prospected in order to provide new ideas for the further study of natural organic polymer coagulants.
\end{abstract}

\section{Introduction}

The coagulation sedimentation method is one of the most common operation units in water treatment process, which adds coagulant to water to make colloidal and suspended particles aggregate into larger particles and separate them from water to achieve the purpose of water purification. This method has attracted much attention because of its simple operation, economic and effective characteristics ${ }^{[1-2]}$. Coagulant selection is the core of coagulation process. Compared with inorganic and synthetic coagulants, natural organic polymer coagulants have become research hotspot at home and abroad since 1990s because of their many active groups, wide source of raw materials, low price, safety, nontoxicity and good biodegradability advantages ${ }^{[3-6]}$. However, the application and commercialization of biodegradable coagulants were limited by the loss of coagulant activity and the instability of coagulants ${ }^{[7-10]}$. In this paper, the main types of the natural organic ploymer coagulants are summarized, the research progress of the stability of coagulants are expounded, and the application in water treatment is also discussed. Finally, the future development directions of coagulants are prospected in order to provide a new reference for the further study of natural organic polymer coagulants.

\section{Classification of natural organic polymer coagulants}

Natural organic polymer coagulants refer to a product obtained by extracting or modifying an organic polymer substance in agricultural by-products in nature ${ }^{[11]}$. Due to the abundant sources of raw materials, there are many kinds of natural organic polymer coagulants. Among them, the following four types are widely used.

\subsection{Starch coagulant}

Starch is one of the most abundant natural ploymer biopolysaccharides in the world ${ }^{[12]}$. Its content in nature is much higher than other organic substances. It is the most abundant organic resources available to human beings, such as wheat, corn, potatoes and rice. Starch segment is an ideal natural organic polymer coagulation material because it contains a large number of active groups - hydroxyl groups (-OH) and has strong reactivity.

\subsection{Chitosan coagulant}

Chitosan coagulant is a linear polymer with molecular weight over $10^{5}$ obtained by deacetylation of chitin, which widely exists in insects and crustaceans (shrimp, crab, etc.). Because of its remarkable versatility and biocompatibility, it has become a new type of green coagulant material, which can effectively remove organic substances and heavy metals in water, so it is widely used in water treatment.

\subsection{Lignin coagulant}

Lignin is a natural resource rich in natural minerals. When lignin is used as coagulant, its average molecular weight is low and its active adsorption point is small. Generally, the spatial structure of lignin is changed by crosslinking reaction and condensation reaction, and its molecular weight is increased to improve the coagulation performance of lignin ${ }^{[13]}$.

\subsection{Plant gum coagulant}

\footnotetext{
* Corresponding author: cea_fuy@ujn.edu.cn
} 
Plant gum is a natural polysaccharide ploymer compound extracted from plant or plant seeds. Its main component is galactomannan. Its molecular weight varies from source to source. Galactose mannose not only has abundant active groups, but also has good water solubility and cross-linking properties. It can form high viscosity aqueous solution at low concentration, so it has significant development value in the field of water treatment ${ }^{[14]}$

\section{Stability of natural organic polymer coagulants}

Stability is one of the indexes to measure the excellent properties of coagulants and one of the characteristics affecting the application of coagulants. Starch and lignin natural organic ploymer coagulants developed rapidly in the 1970s and 1980s. However, due to their poor stability, the research process of starch and lignin natural organic ploymer coagulants have been at a low ebb for a long time. In recent years, with people's desire for good environmental quality, it is imperative to develop green and environmental friendly coagulants. At the same time, the research and development of natural organic ploymer coagulant has opened up a new way for the utilization of natural resources ${ }^{[15-17]}$. In addition, from the domestic and international situation, the development of natural organic ploymer coagulants also make stability problem more and more attention.

\subsection{Unstable performances}

W.X. Jiang et al. ${ }^{[18]}$ studied the stability of natural organic ploymer coagulants CCNF-2 and RHNF-2 prepared from corn cob powder or rice husk powder, and tested their coagulation effect with time.The experimental results show that the coagulation effect decreases gradually with the passage of time. The coagulation effect of CCNF-2 coagulant declined rapidly after 5 days, RHNF-2 coagulant declined rapidly after 7 days and almost disappeared after 15 days. It shows that the stability of coagulant really needs to be improved.

S. Wang et al. ${ }^{[3]}$ found that a coagulant prepared from Koelreuteria paniculata seeds water extract had an unpleasant odor after standing at high temperature for about two days, indicating that microorganisms had begun to decompose proteins and other organic substances and had a degradation reaction. It can be concluded that although good degradability is beneficial to the environment, it also weakens the coagulation performance of Koelreuteria paniculata seeds water extract. This study shows that temperature is one of the factors affecting the stability of water extract, because it accelerates the production of microorganisms in water extract, which leads to poor stability.

It can be known from classification above, natural organic polymer coagulants contain a large amount of organic substances such as saccharides, which are composed of active groups such as carboxyl groups ($\mathrm{COOH})$ and hydroxyl groups $(-\mathrm{OH})$, these active groups are the key to coagulation activity. Natural organic polymer coagulants in liquid state is equivalent to liquid medium having a suitable condition, which is easy to produce microorganisms. These microorganisms decompose organic matter and degrade coagulation components, resulting in unstable coagulation effect. It was found that the stability of coagulation effect was related to microorganisms.

Fig. 1 shows microbial changes in the natural organic polymer coagulant. The changes of microorganisms in the coagulant were observed by Olympus CX31 optical microscope $(10 \times 10)$.

As can be seen from Fig. 1, with the passage of time, the microorganisms in the liquid natural organic polymer coagulant are increasing. At $0 \mathrm{~d}$ (the day of preparation of coagulant), only a few microorganisms appeared. At 1 $\mathrm{d}$, the coagulant deteriorated and observed that the number of microorganisms increased and aggregated. At $7 \mathrm{~d}$, compared with previous days, the number and size of microorganisms aggregation were more and more. This indicates that the stability of the natural organic polymer coagulant is not good. The phenomenon of microorganisms production is similar to S. Wang's study $^{[3]}$ when he studied Koelreuteria paniculata seeds water extract coagulant.

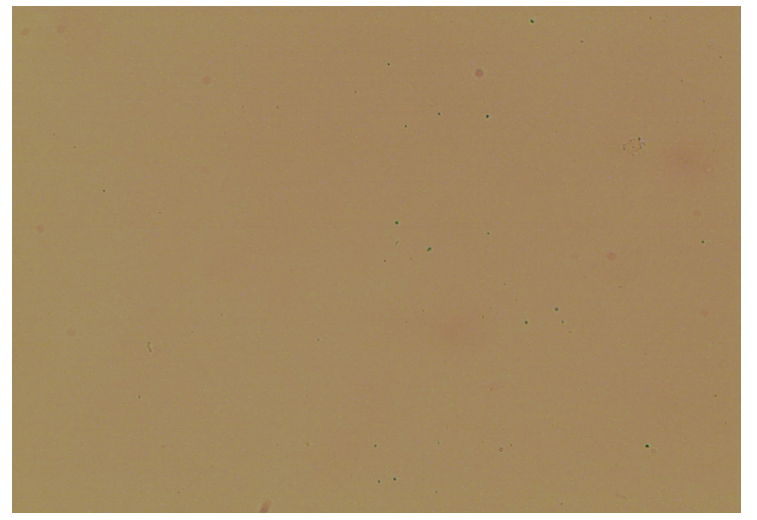

(a) $0 \mathrm{~d}$

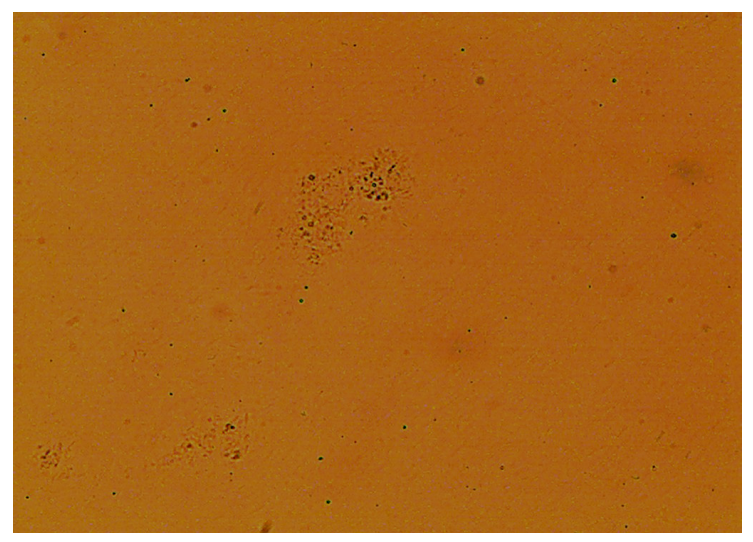

(b) $1 \mathrm{~d}$ 


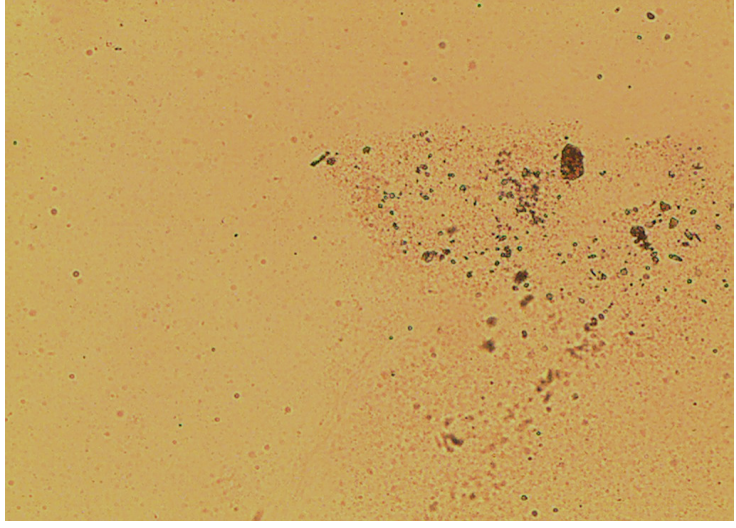

(c) $7 \mathrm{~d}$

Fig. 1. Microbial changes in the natural organic polymer coagulant at different times

\subsection{Stability improvement measures}

R.F. Jin et al. ${ }^{[4]}$ studied that natural coagulant had good coagulation activity and stability during 100 days of storage at $4{ }^{\circ} \mathrm{C}$ and room temperature. After 100 days, the coagulation effect decreased rapidly. After 140 days, the coagulation activity of the coagulant placed at room temperature was lower than that of the coagulant stored at $4{ }^{\circ} \mathrm{C}$. In addition, $40 \%$ polysaccharide, $35 \%$ protein and $10 \%$ nucleic acid were found in the coagulant. This experiment showed that cold storge $\left(4^{\circ} \mathrm{C}\right)$ could helpful to improve the stability of natural organic polymer coagulants. N. Zhang et al. ${ }^{[19]}$ placed the coagulant produced by aspergillus sojae at a low temperature of $4{ }^{\circ} \mathrm{C}$ for 35 days, and the coagulation rate was still above $96 \%$, while the coagulation rate of biocoagulant at different $\mathrm{pH}$ values after 35 days of storage at room temperature was very different.

J. Wang et al. ${ }^{[20]}$ used cactus gel to prepare the natural ploymer coagulant. When the dosage was $1 \mathrm{mg} / \mathrm{L}$ to $6 \mathrm{mg} / \mathrm{L}$, the removal rate of turbidity of kaolin suspension reached $99.3 \%$. Compared with other coagulants, the dosage was less. The cactus gel and sulphuric acid solution were made at 1:10. After 1 weeks' storage, the coagulability of the cactus gel decreased little. This shows that the cactus gel processed by this method can maintain good stability within a week.

H.H. Guo et al. ${ }^{[21]}$ studied the stability of aloe coagulant and found that low temperature and low $\mathrm{pH}$ could improve its stability. Aloe gel can be refrigerated for more than 18 days after being treated with potassium sorbate, citric acid $\mathrm{pH}$ and activated carbon adsorption. At the same time, the results showed that after 14 days' stabilization, the coagulant effect remained above $90 \%$ and maintained good coagulating activity. X.Y. $\mathrm{Jia}^{[22]}$ found that only chitosan had poor stability. However, the preparation of composite chitosan coagulant from fly ash can improve the stability of wastewater treatment and remove phosphorus from wastewater.

\section{Application of natural organic polymer coagulants}

Natural organic polymer coagulants are widely used in water supply and wastewater treatment, such as Yangtze river water, drinking water, domestic sewage, heavy metal ion wastewater, printing and dyeing wastewater and sludge conditioning, because of their safety, nontoxicity, abundant raw materials and low cost ${ }^{[23]}$.

Y.H. Luo ${ }^{[24]}$ used cactus as raw material to prepare coagulant for domestic sewage and Yangtze river water. The removal rate of $\mathrm{COD}_{\mathrm{cr}}$ is $60 \%$ when domestic sewage is treated, which is better than that of aluminium chloride hexahydrate. In addition, although the coagulation effect of cactus coagulant on Yangtze river water is slightly worse than that of inorganic coagulant, it produces flocs with high speed, large particle size and fast settling speed. It shows that the addition of cactus coagulant is helpful to the subsequent water treatment process.

J. Li et al. ${ }^{[25]}$ treated citric acid sludge with chitosan and PAM respectively. The results of comparison experiments showed that the optimal dosages of chitosan and PAM were 17.0 and $8.4 \mathrm{mg} / \mathrm{g}$, respectively, after $1900 \mathrm{r} / \mathrm{min}$. Centrifugation, when the centrifugation time is $>7 \mathrm{~min}$, the chitosan-treated citric acid sludge has a higher solid content than the PAM-treated sludge, ie chitosan to citric acid sludge The dehydration effect is better than PAM. Scanning electron micrographs of citric acid sludge flocs treated with chitosan and PAM showed that the chitosan-treated citric acid sludge was smaller than the PAM-treated sludge flocs and the pores between the flocs. More, this is the main reason why chitosan has better treatment effect on citric acid sludge than PAM.

M.F. Pistonesi et al. ${ }^{[26]}$ treated drinking water with modified chitosan coagulant. When the dosage was 10.5 $\mathrm{mg} / \mathrm{L}$, the turbidity decreased by $99 \%$, COD decreased by $75 \%$, and various algae could be removed. S.Y. Jiang et al. ${ }^{[27]}$ developed a kind of chitosan coagulant CAF. It was found that CAF has obvious purification effect on tap water with turbidity removal rate of $99.6 \%$, and can effectively inhibit bacterial growth and remove harmful substances such as chlorine in water.

S.B. Lalvani et al. ${ }^{[28]}$ treated the aqueous solution containing $\mathrm{Cr}^{6+}$ and $\mathrm{Cr}^{3+}$ with lignin coagulant, the removal rate of $\mathrm{Cr}^{6+}$ was $63 \%$, and the removal rate of $\mathrm{Cr}^{3+}$ was $100 \%$.

Q. Zhang et al. ${ }^{[29]}$ synthesized dimethylpropenyl lignin quaternary ammonium salt (DL) coagulant from lignin in black liquor of papermaking. The experimental results showed that the decolorization effect of DL coagulant on acid black ATT dye reached $80 \%$ under the optimum conditions $\left(30^{\circ} \mathrm{C}\right.$ and $\left.\mathrm{pH}=1.5\right)$. G.V. Duarte et al. ${ }^{[30]}$ extracted lignin from hardwood under hot water conditions at $160{ }^{\circ} \mathrm{C}$ to prepare nano-scale cationic lignin polymer coagulant, which has a coagulation effect of more than $90 \%$ on printing and dyeing wastewater. It can be seen that lignin coagulant is a good choice for treating printing and dyeing wastewater.

F. Ren et al. ${ }^{[31]}$ used orange slag and pineapple slag as raw materials to prepare a variety of coagulants, which were used to treat papermaking wastewater, which could reduce the suspended solids and COD of 
papermaking wastewater by $60 \%$ and $50 \%$ above, respectively.

L.Q.Z. Li et al. ${ }^{[32]}$ prepared a king of coagulant using waste chili residue as raw material. It was found that the coagulant had good coagulation performance for domestic sewage under alkaline conditions. Y.J. Chen ${ }^{[33]}$ used the agricultural waste mulberry to prepare a coagulant for the treatment of landscape water, and the COD removal rate reached $78.3 \%$.

R. Divakaran et al. ${ }^{[34]}$ found that the treatment of sludge in river water with chitosan coagulant can effectively reduce the turbidity caused by sludge, and the flocs formed in the treatment process are fibrous and large in volume. D.Z. Lu et al. ${ }^{[35]}$ found that the oil removal rate was $86.7 \%$ and the residual oil in water was only $8 \mathrm{mg} / \mathrm{L}$ by using grafted starch coagulant to treat petroleum wastewater.

\section{Development trends and prospects}

The development of coagulants in China has gradually changed from low molecular to polymer, inorganic to organic, single to composite, and more environmentally friendly ${ }^{[36]}$. After entering the 21st century, the development of green industry has become the focus of human attention. Natural organic polymer coagulants reflect the environmental protection concept of "turning waste into treasure". The research of natural organic polymer coagulants is the inevitable result of conforming to the trend of the times ${ }^{[37]}$.

In the future, research on natural organic polymer coagulants mainly has the following aspects ${ }^{[38-41]}$ :

(1)To further improve the stability of existing natural organic polymer coagulants;

(2)Study on modified natural organic polymer coagulants and develop organic polymer coagulants using new natural materials;

(3)Broaden the application scope of natural organic polymer coagulants.

\section{References}

1. L.Y. Jiang, H. Liu, X.L. Zhu, X.Y. Fan, Environ. Sci. Technol. 36, 128-133 (2013).

2. X. Huang, B.Y. Gao, Y. Wang, Q.Y. Yue, Q. Li, Y.Y. Zhang, Chem. Eng. J. 245,173-179 (2013).

3. S. Wang, Shanxi University of Science and Technology, Xi'an, (2014).

4. R.F. Jin, J. Wang, J.T. Zhou, C.Y. Wang, T.C. Cao, China Environ. Sci. 28, 881-891 (2008).

5. Z.H. Lin, M.G. Li, Technology of Water Treatment, 29, 315-317 (2003).

6. Y.M. Wang. Sichuan Environ. 28, 71-74 (2009).

7. C. Nakason, T. Wohmang, A. Kaesaman, S. Kiatkamjornwong, Carbohydr. Polym. 81, 348-357 (2010).

8. M. Huang, University of Nanjing, Nanjing, (2016).

9. J.P. Chen, B. Zhu, J.F. Zhang, Technology of Water Treatment, 40, 6-10 (2014).
10. Z.Y. Liu, K. Hu, B. Dai, J.J. Lu, J. Shihezi Univ. (Nat. Sci.), 26, 175-179 (2008).

11. N.N. Lu, University of Jinan, Jinan, (2017).

12. H. Song, D. Wu, R.Q. Zhang, L.Y. Qiao, S.H. Zhang, S.Lin, J. Ye, Carbohydr. Polym. 78, 253-257 (2009).

13. Y. Zhao, Y.M. Li. Leather Chemicals 24, 26-30 (2007).

14. P. Ren, X.W. Ruan, D.L. Wang, Food Research and Development, 25, 39-44 (2004).

15. Z. Yang, H. Wu, B. Yuan, M. Huang, H.Yang, A. Li, Chem. Eng. J. 244, 209-217 (2014).

16. X. Jiang, Y.J. Qi, S.G. Wang, X.Z. Tian, J. Hazard. Mater. 173, 298-304 (2010).

17. Q.Y. Zhou, W. Guo, Y. Xiao, F.W. Meng, Guangzhou Chem. Ind. 41, 50-52 (2013).

18. W.X. Jiang, Tianjin University of Science and Technology, Tianjin, (2015).

19. N. Zhang, Microbiol. China, 35, 685-689 (2008).

20. J. Wang, Nanjing University of Science and Technology, Nanjing, (2008).

21. H.H. Guo, Nanjing University of Science and Technology, Nanjing, (2008).

22. X.Y. Jia, University of Chongqing, Chongqing, (2004).

23. J.J. Liu, Y. Kang, Chem. Ind. Eng. 22, 476481(2005).

24. Y.H. Luo, University of Wuhan, Wuhan, (2005).

25. J. Li, W.R. Hu, H.Y. Pei, Industrial Water Treatment, 28, 18-21 (2008).

26. M.F. Pistonesi, M.S. Rodriguez, E. Agullo, Adrances in Chitin Science, 165-170 (2000).

27. S.Y. Jiang, T.Y. Gao, W.J. Zhang, Environmental Protection of Chemical Industey, 20, 32-35 (2000).

28. S.B. Lalvani, A. Hubner, T.S. Wiltowski. Energy Sources, 22, 45-46 (2000).

29. Q. Zhang, S.X. Ren, Y.L. Ma, G.Z. Fang, J. Funct. Mater. 45, 138-141 (2014).

30. G.V. Duarte, B.V. Ramarao, T.E. Amido, Bioresour. Technol. 101, 8526-8534 (2010).

31. F. Ren, X.X. Li, X.Y. Zhou, Y. Zhang, Fujian Environ. 20, 24-26 (2003).

32. L.Q.Z. Li, Y. Deng, J.M. Feng. Technology of Water Treatment, 32, 66-68 (2006).

33. Y.J. Chen, Jiangsu University of Science and Technoiogy, Zhenjiang, (2009).

34. R. Divakaran, V.N.S. Pillai, Water Res. 38, $2135-$ 2143 (2004) .

35. D.Z. Lu, G.S. Wu, Y.M. Hu, M.X. Li, G.C. Yue, Z.M. Yang, J. Jilin Univ.(Nat. Sci. Ed.), 8185(1989).

36. X.X. Yu, X.L. Pu, Environmental Protection of Oil \& Gas Fields, 17, 43-46 (2007).

37. J.H. Wang, J.J. Xu, S. Wang, Z.H. Lv, Paper and Paper Making, 36, 34-37(2017). 
38. X.X. Cao, J.G. Xiong, S.M. Chen, C.B. Shao, Journal of Chongqing Technology and Business University (Nat. Sci. Ed.), 28, 635-638 (2011).

39. M.J. Liu, H.R. Wang, China Leather, 47, 36-40 (2018).

40. C.L. Lin, Dalian Polytechnic University, Dalian, (2012).

41. Y. Wei, M. Huang, A.M. Li, H. Yang, Environ. Chem. 37, 1293-1310 (2018). 